\title{
AUTOMATIC DETECTION OF BURNED AREAS IN WETLANDS BY REMOTE SENSING MULTITEMPORAL IMAGES
}

\author{
Daniel Capella Zanotta ${ }^{1,2}$, Hiran Zani ${ }^{1}$, Yosio Edemir Shimabukuro ${ }^{1}$ \\ ${ }^{1}$ National Institute for Space Research - São José dos Campos - SP - Brazil \\ ${ }^{2}$ National Institute for Science, Education and Technology - Rio Grande - RS - Brazil
}

\begin{abstract}
In this paper, a methodology for automatic detection of burned areas is suggested. The classification criterion is performed using Bayesian statistical parameter (mean and covariance matrix) extracted automatically using the Expectation Maximization algorithm and taking into account the spectral similarity between burned and flooded areas. In this work the final process involves the application of morphological operators of erosion and dilation of images in order to insert information from the spatial context, refining the final map. Experiments were conducted to a TM-Landsat scene with areas affected by fires and seasonal flooding. The results show that the accuracy is increased with the consideration of flooding mask and the subsequent application of spatial context, reaching values up to $97 \%$ of accuracy when compared with a reference map.
\end{abstract}

Index Terms - Optical imagery, Land surface change, Pattern recognition

\section{INTRODUCTION}

Change detection through remote sensing imagery has been widely studied by many researchers in recent decades [1], [2], [3]. One of the most widely used techniques is based on the "difference image" [1], where data from images acquired at two different times are subtracted element by element (which can be pixels or objects). The differences can be computed either from the original features or from features extracted from the original data, such as principal components or vegetation indices. Usually, the classification between changed and non-changed elements is performed by using empirical strategies or manual trial-and-error procedures, which affect both the accuracy and the reliability of the change detection process [2]. Some studies propose a classification based on the Bayes theory, in which the changes are identified by estimating the parameters of the statistical distribution for 'change' and 'no-change' classes [2], [4]. In wetlands, the automatic detection of burned areas finds a problem caused by confusion with changes in hydrology: the similarity between the spectral and spatial characteristics of moist soil and burned areas constitute a major source of error [5], [6]. In this paper, we present a methodology to accurately detect burned areas in wetlands based on the Bayesian theory while avoiding unpredictable and rapid changes caused by flooding.

\section{PROPOSED APPROACH}

Following most change detection studies, the first step of our proposed approach consists of producing the difference image between two dates analyzed by subtracting pixel by pixel each of the respective available image multispectral channels. The resulting difference image shows the changes occurring between the two dates. In order to classify each pixel $(X)$ of the image in one of the two exhaustive classes (change or no-change) by means of the Bayes theory, we need to define the statistical parameters of the two classes. In this study we employ the Expectation Maximization algorithm (EM), as described by [7], to automatically estimate the means vector, covariance matrix and the $a$ priori probabilities for both classes. The rationale of using the EM algorithm to this end relies on the fact that both classes (change and no-change) tend to occur according a normal distribution with different parameters in the difference image. The output data computed by the EM algorithm are used as the input in a Bayesian classifier assuming a multivariate normal distribution:

$G_{i}(X)=(2 \pi)^{-p / 2}\left|\Sigma_{i}\right|^{-1 / 2} \exp \left[1 / 2\left(X-\mu_{i}\right)^{\Sigma_{i}^{-1}}\left(X-\mu_{i}\right)\right] P\left(\omega_{i}\right)$ where $G_{i}(X)$ is the decision function for each class of change $(i), p$ is the data dimensionality, in this case $p=2, \Sigma_{i}$ is the covariance matrix, $\mu_{i}$ the means vector and $P\left(\omega_{i}\right)$ the a priori probabilities of each class. Considering classes $\omega_{1}$ (change) and $\omega_{2}$ (no change) the Bayesian rule is given by:

$$
\text { if } G_{1}(X)>G_{2}(X), \quad \text { then: } X \in \omega_{1}
$$

(change) ; otherwise: $X \in \omega_{2}$ (no-change). 


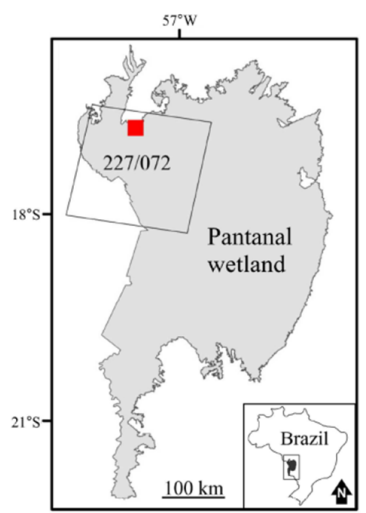

(a)

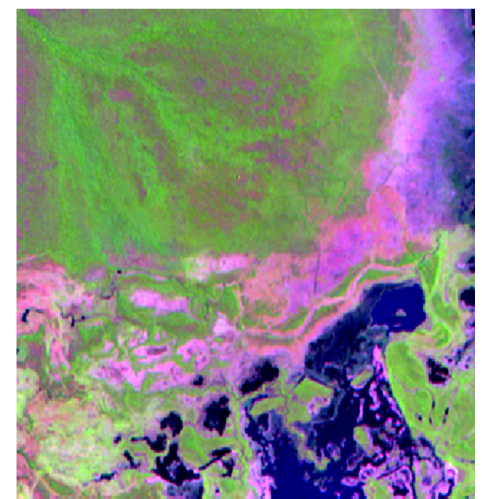

(b)

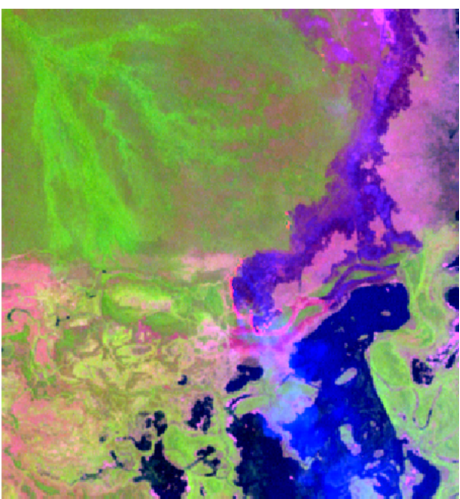

(c)

Fig. 1. (a) Location of study area, (b) 31/Aug/2001 image acquired before fire events, and (c) 05/Sep/2002 image acquired after fire events. (b) and (c) multispectral compositions: 5(R)4(G)3(B).

After this process, a binary change map is generated with digital numbers of 0 (i.e., black) representing change areas and 255 (i.e., white) representing nochange areas. It is worth noting that, for wetlands marked by fire events, which are our subject of study, the changes will initially be associated to burned areas as well as flooded areas. In order to exclude water bodies and moist soils from the subsequent change map, a mask is obtained using a reflectance threshold of $0-0.5 \%$ in the channel TM-Landsat 5 . This mask is then used to exclude the water bodies from the change map. Contextual information can be finally applied by means of morphological operators of erosion and dilatation of images [8] to eliminate the not significant or noise-like changes. This method was chosen because of its effectiveness in eliminating small areas corresponding to changes that are not of interesting to this study. Given the dynamic of Pantanal region, these small changes are common.

\section{EXPERIMENTS AND RESULTS}

To illustrate the proposed methodology we performed an experiment with a TM-Landsat 5 image as date 1 acquired on August 2001 (Fig. 1.b), and as date 2 acquired on September 2002 (Fig. 1.c). The study area is located in the central portion of South America inside the Brazilian Pantanal (Fig. 1a), which is a vast wetland associated with active tectonics and fluvial sedimentation [9]. This area is ideal to test the proposed methodology because there is a high occurrence of fires and seasonal flooding. A large fire event and flooding episodes were registered between image acquisitions. The scenes were provided by the U.S. Geological Survey [10], both had a Level-1 Terrain (L1T) degree of correction and were orthorectified with an RMS error lower than 0.8 pixel [10]. All images had their digital number converted to exoatmospheric reflectance values according to a procedure described in [11] with updated calibration coefficients. Finally, radiometric normalization was applied using the dark object subtraction method [12], which is sufficient for the goals of the present study.

In the Fig. 2.a we can see the results produced by the Bayesian classification and the improvement reached by the exclusion of water bodies (Fig. 2.b). The resulting effect caused by the application of spatial context information by morphological operators with 15 iterations can be seen in Fig. 2.c. As expected, the initial change map (Fig. 2.a) shows artifacts, mostly caused by water bodies and moist soil. However, the exclusion of water bodies and the subsequent application of simple morphological operators considerably improved the initial results. A quantitative assessment by means of overall accuracy based on a manually produced reference map showed that a substantially reduction in post-classification errors was achieved with the combination of Bayesian classification based on statistical parameters estimated by EM algorithm, subsequent exclusion of the water bodies, and the application of spatial context information by morphological operators (Table 1). An iteration-by-iteration comparison between the reference image and the change map was performed with results showing the best result in the fifteenth iteration (Fig. 3). 


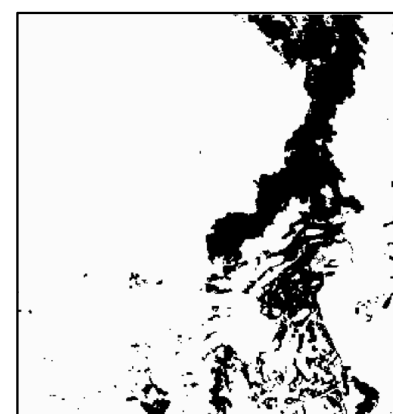

(a)

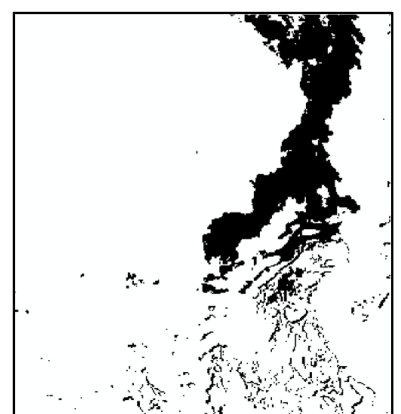

(b)

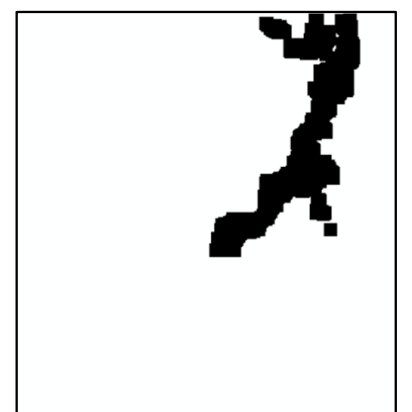

(c)

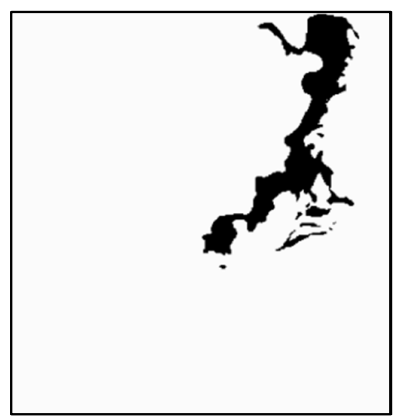

(d)

Fig. 2. Classification maps obtained with the proposed methodology. (a) Bayesian classification with statistical parameters based on EM algorithm; (b) Bayesian classification considering the water exclusion mask; (c) after application of morphological operators with 15 interactions; and (d) reference map.

Table 1. Quantitative assessment of results.

\begin{tabular}{|c|c|c|c|}
\hline $\begin{array}{c}\text { Burn detection } \\
\text { method }\end{array}$ & $\begin{array}{c}\text { Correctly } \\
\text { classified } \\
\text { pixels }\end{array}$ & $\begin{array}{c}\text { Incorrectly } \\
\text { classified } \\
\text { pixels }\end{array}$ & $\begin{array}{c}\text { Overall } \\
\text { accurac } \\
\text { y }\end{array}$ \\
\hline Classified image & 93232 & 10997 & $89,4 \%$ \\
\hline $\begin{array}{c}\text { Classified image }+ \\
\text { Water body mask }\end{array}$ & 96704 & 7525 & $92,7 \%$ \\
\hline $\begin{array}{c}\text { Classified image }+ \\
\text { Water body mask }+ \\
\text { Morph. operators } \\
\text { after 15 iterations }\end{array}$ & 101206 & 3023 & $97,1 \%$ \\
\hline
\end{tabular}

The solid line in Fig. 3 represents the performance of the morphological operators applied directly in the Bayesian classification, and the dashed line is the performance considering the water mask. As can be seen, the process became stable between the10th and 20th iterations. This allows us to suggest that the ideal number of iterations lies in a range of $10-20$. It is worth noting that after the 15th interaction, the solid and dashed lines became overlapped. This result can be explained by the fact that, by the time the number of iterations increases, even in large regions, such as those resulting from flooding, the water features are eliminated from the map. However, it depends on the size of the water bodies present in the scene, i.e. when the size of the flooded regions is larger, the number of iterations needed until the lines overlap is greater. The time for this effect to occur is proportional to the size of the flood-labeled features. According to Fig. 3, after a certain number of iterations, the accuracy of the method begins to decline; therefore, the preelimination of the flood-labeled regions is an important factor to the quality of the final results since it can reduce the number of iterations.

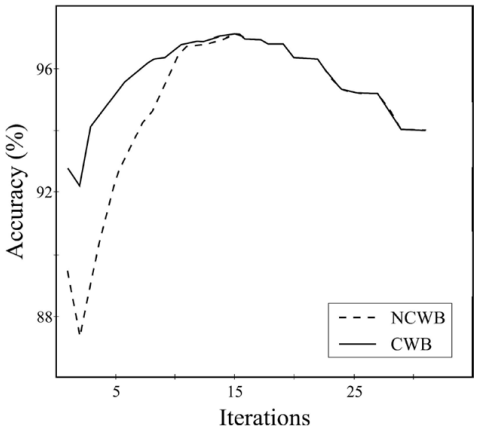

Fig. 3 Accuracy as a function of the number of interactions, considering the water bodies (CWB) and not considering the water bodies (NCWB).

\section{CONCLUSIONS}

In this work we suggested a new methodology to automatically extract burned areas in wetlands from remote sensing multitemporal images. Our results support that a Bayesian classification followed by the exclusion of water bodies and the addition of spatial context information by morphological operators were effective to detect the burned areas in the study area. Some care needs to be taken concerning the number of iterations of the morphological operator. Despite the fine results reached by classification, the proposed methodology needs to be tested with other areas to be considered systematically. 


\section{REFERENCES}

[1] A. Singh, "Digital change detection techniques using remotelysensed data", International Journal of Remote Sensing, vol. 10, no 6, pp. 989-1003, 1989.

[2] L. Bruzzone and D.F. Prieto, "Automatic analysis of the difference image for unsupervised change detection". IEEE Transactions on Geoscience and Remote Sensing, vol. 38, no. 3, pp. 1171-1182, 2000.

[3] B. Demir, Bovolo, F. Bruzzone, L., "Detection of Land-Cover Transitions in Multitemporal Remote Sensing Images with Active Learning Based Compound Classification", IEEE Transactions on Geoscience and Remote Sensing, vol. 50, no. 5, pp. 1930-1941, 2012.

[4] D.C. Zanotta and V. Haertel, "Gradual land cover change detection based on multitemporal fraction images", Pattern Recognition, vol. 45, n. 8, pp. 2927-2937, 2012.

[5] Huete, A.R., Jackson, R.D. and Post, D.F., "Spectral response of a plant canopy with different soil backgrounds". Remote Sensing of Environment, vol. 17, pp. 37-53, 1985.

[6] G.H. Mitri, and I.Z. Gitas, "A semi-automated object-oriented model for burned area mapping in the Mediterranean region using Landsat-TM imagery". International Journal of Wildland Fire, vol. 13, pp. 367-376, 2004.

[7] R.O. Duda, P.E. Hart, D.G. Stork, 2001, Pattern Classification, 482 p. (New York: John Wiley \& Sons).

[8] R.A. Schowengerdt, 2006, Remote sensing: models and methods for image processing. (New York: John Wiley \& Sons)

[9] M.L. Assine, and P.C. Soares, "Quaternary of the Pantanal, west-central Brazil”. Quaternary International, vol. 114, pp. 2334, 2004.

[10] USGS, 2009, Landsat product type descriptions. Available online at: http://edcsns17.cr.usgs.gov/helpdocs/landsat/product_ descriptions.html (accessed 04 September 2010).

[11] G. Chander, and B.L. Markham, "Revised Landsat-5 TM radiometric calibration procedures and post calibration dynamic ranges". IEEE Transactions on Geoscience and Remote Sensing, vol. 41, no. 11, pp. 2674-2677, 2003.

[12] P. Chavez, "An improved dark-object subtraction technique for atmospheric scattering correction of multispectral data". Remote Sensing of Environment, vol. 24, no. 3, pp. 459-479, 1988. 